\title{
CUANTIFICACIÓN DE ANTOCIANINAS MEDIANTE ESPECTROSCOPÍA DE INFRARROJO CERCANO Y CROMATOGRAFÍA LÍQUIDA EN MAÍCES PIGMENTADOS
}

\author{
QUANTIFICATION OF ANTHOCYANINS THROUGH NEAR INFRARED SPECTROSCOPY \\ AND LIQUID CHROMATOGRAPHY IN PIGMENTED MAIZE
}

\author{
Juan de Dios Hernández-Quintero', 2, Aldo Rosales-Nolasco², Aidé Molina-Macedo², \\ Alejandra Miranda-Piliado ${ }^{2}$, Martha Willcox ${ }^{2}$, Juan M. Hernández-Casillas ${ }^{3}$ y Natalia Palacios-Rojas ${ }^{2 *}$
}

\begin{abstract}
'Postgrado en Recursos Genéticos y Productividad - Genética, Campus Montecillo, Colegio de Postgraduados. km 36.5 Carr. México-Texcoco. 56230, Montecillo, Texcoco, Estado de México. ${ }^{2}$ Centro Internacional de Mejoramiento de Maíz y Trigo. km 45 Carr. México-Veracruz. El Batán. 56130 Texcoco, Edo. de México. ${ }^{3}$ Programa de Recursos Genéticos, Campo Experimental Valle de México, Instituto Nacional de Investigaciones Forestales,
\end{abstract} Agrícolas y Pecuarias. km 13.5 Carr. Los Reyes-Texcoco. 56250, Coatlinchán, Texcoco, Estado de México, México.

*Autor para correspondencia: n.palacios@cgiar.org

\section{RESUMEN}

El método de espectroscopia de infrarrojo cercano se utiliza ampliamente para la determinación de ciertos compuestos químicos. Actualmente es una herramienta de soporte para los programas de mejoramiento, que permite la determinación de compuestos como proteína, almidón, aceite, humedad y cenizas, entre otros. Un total de 554 muestras pertenecientes a 24 razas de maíz (Zea mays L.) pigmentado fueron utilizadas en el desarrollo y validación de modelos matemáticos para la estimación del contenido de antocianinas por espectroscopía de infrarrojo cercano (NIR), a través de la espectroscopía de UV-Vis como método de referencia. Se determinó el perfil de antocianinas mediante cromatografía líquida de alta resolución (HPLC). Los más altos contenidos de antocianinas se encontraron en muestras de grano de maíz C11-IXT de Tlaxcala, México, con hasta $1989.9 \mu \mathrm{g}$ Pel g ${ }^{-1}$ PS. Los mayores porcentajes de cianidina 3-glucósido, pelargonidina 3-glucósido y peonidina 3-glucósido fueron $48.79 \%, 39.84 \%$ y $12.14 \%$, respectivamente. Muestras de la accesión BOZM342 destacaron por el contenido de cianidina 3-glucósido, que fue de $628.32 \mu \mathrm{g} \mathrm{g}^{-1}$ PS. Se desarrollaron y analizaron 32 modelos de calibración de los cuales destacaron dos por cumplir los parámetros para una calibración de NIR robusta, con altos coeficientes de determinación para las validaciones cruzadas (0.64 y 0.65). Los modelos de NIR presentados en este trabajo se pueden utilizar para la determinación de antocianinas totales y apoyar a los programas de mejoramiento de maíces azules. NIR

Palabras clave: Zea mays, antocianinas, HPLC, maíz pigmentado,

\section{SUMMARY}

Near infrared reflectance, NIR, is a method largely used for rapid and robust determination of chemical compounds. It is currently used to support breeding programs to analyze protein, oil, starch, moisture and ash content, among others. Five hundred fifty four samples belonging to 24 races of pigmented maize (Zea mays L.) were used in the development and validation of mathematical models to estimate anthocyanin content by near-infrared spectroscopy (NIR), using UV-Vis spectroscopy as a reference method. The anthocyanins profile was determined through high performance liquid chromatography (HPLC). The highest anthocyanin contents were found in C11-IXT maize grain samples from Tlaxcala, México; containing up to $1989.97 \mu \mathrm{g} \mathrm{Pel} \mathrm{g-1} \mathrm{DW.} \mathrm{The} \mathrm{highest}$ percentages of cyanidin 3-glucoside, pelargonidin 3-glucoside and peonidin 3-glucoside were $48.79 \%, 39.84 \%$ and $12.14 \%$, respectively. Samples of the
BOZM342 accession were outstanding for its cyanidin 3-glucoside content of $628.32 \mu \mathrm{g} \mathrm{Pel} \mathrm{g-1}$ PS. Thirty two calibration models were developed and analyzed, and two of them fulfilled the parameters for a robust NIR calibration, with high coefficients of determination for cross validations ( 0.64 and 0.65 ) The NIR models presented here can be used for determination of total anthocyanins and provide support for blue maize breeding programs.

Index words: Zea mays, anthocyanins, HPLC, pigmented maize, NIR.

\section{INTRODUCCIÓN}

En México, centro de origen y diversidad del maíz (Zea mays L.), existen 64 razas de maíz y 23 de ellas tienen variantes de grano pigmentado (CONABIO, 2016). Dentro de las variantes en el color de grano de los maíces pigmentados se encuentran desde el negro, café, rojo hasta rosa pálido, y los maíces rojos y azules son los más comunes (Salinas et al., 2013). Los maíces pigmentados son apreciados por los consumidores debido al color, textura y sabor que imparten al producto procesado, y por lo tanto son utilizados en la preparación de diversos platillos típicos como atoles, tortillas, pozole, totopos, etc. (Fernández et al., 2013). Estos maíces son considerados especiales y, aunque la producción alcanzó solamente 12,916.1 t en el año 2015 (SIAP, 2017), constituyen una fuente de ingreso importante para agricultores en ciertas regiones del país (Hellin et al., 2013). El color rosado, azul, morado y negro de estos maíces está dado por la presencia de antocianinas, las cuales se acumulan principalmente en el pericarpio y la capa de aleurona (Agama-Acevedo et al., 2011). Las antocianinas del tipo cianidina y malvidina se encuentran en maíces azules, morados y negros, mientras que los maíces más rosados contienen pelargonidina, cianidina y malvidina (Salinas-Moreno et al., 2003).

Las antocianinas son compuestos sintetizados por las 
plantas y se clasifican como flavonoides, que se acumulan en cualquier órgano vegetativo en respuesta a radiación ultravioleta. Su consumo en la dieta humana disminuye el riesgo de padecer enfermedades cardiacas, diabetes, artritis, cáncer, y destaca su alto potencial antioxidante y antiinflamatorio (Hosoda et al., 2012; Zhang et al., 2005). Dada su alta biodisponibilidad, las antocianinas tienen gran aplicación en productos nutracéuticos y terapéuticos en la industria alimenticia y farmacéutica (Ananga et al., 2013).

Existen diferentes métodos de determinación de antocianinas en matrices alimenticias. La espectroscopía de UV-Vis es el método más utilizado para cuantificar antocianinas totales a una longitud de onda de 465 a $550 \mathrm{~nm}$ (He y Giusti, 2010). La cromatografía líquida de alta resolución (HPLC, por sus siglas en inglés) también se utiliza para la cuantificación del perfil de antocianinas; sin embargo, el tiempo de análisis y el costo es mayor comparado con la espectroscopía de UV-Vis. Un método alternativo es la espectroscopía de infra-rojo cercano (NIR, por sus siglas en inglés), el cual permite analizar un gran número de muestras en corto tiempo sin necesidad de hacer extracción previa de los compuestos. Mediante algoritmos quimiométricos se realizan modelos de calibración basados en los datos analíticos obtenidos por los métodos de referencia (Rosales et al., 2011).

El NIR es un método simple, de bajo costo, que permite obtener los resultados en tiempo real, y es de gran utilidad en programas de mejoramiento donde se debe analizar gran número de muestras en periodos de tiempo relativamente cortos. En maíz, el NIR es utilizado para la determinación de proteína, almidón, aceite, humedad, cenizas y determinación de aminoácidos como lisina y triptófano (Rosales et al., 2011). Cavalcanti et al. (2013) desarrollaron un modelo NIR para la determinación de antocianinas en frutos intactos de acai (Euterpe oleracea Mart.) y palmitero-juçara (Euterpe edulis Mart.). Para maíz no se encontraron reportes. Por lo anterior, los objetivos de este trabajo fueron: 1) desarrollar modelos de calibración en el NIR para antocianinas totales y 2) evaluar el perfil de antocianinas en los maíces analizados.

\section{MATERIALES Y MÉTODOS}

\section{Muestras de maíz}

Se utilizaron 254 accesiones de maíz pigmentado, pertenecientes a 24 razas de maíz (Cuadro 1), resguardadas en los bancos de germoplasma del Centro Internacional de Mejoramiento de Maíz y Trigo (CIMMYT) y del Instituto Nacional de Investigaciones Forestales, Agrícolas y Pecuarias (INIFAP). Las accesiones fueron clasificadas en grupos según la altitud de su área de colecta. Los grupos se incrementaron por polinización controlada en ambientes similares a su origen tropical, sub-tropical o valles altos. Se utilizó un diseño sin réplicas de entrada con testigos replicados y se sembraron en Santa Lucía, Edo. de México; Almoloya, Edo. de México; Celaya, Guanajuato; Tarímbaro, Michoacán e Iguala, Guerrero en el ciclo verano-otoño de 2013, según su ambiente de origen. Adicionalmente, y para la validación del método, se utilizaron 26 muestras de maíces adquiridos de agricultores del estado de Tlaxcala (producto de polinización libre 2014). Un total de 554 muestras fueron utilizadas para el desarrollo y validación de los métodos aquí presentados.

\section{Preparación de las muestras}

Con el propósito de desarrollar y validar los métodos analíticos se utilizaron 20 granos al azar por muestra, que fueron molidos en un molino de discos de la marca Perten (Perten, Falling Number 3303, Huddinge, Sweden), con el cual se obtuvieron partículas de aproximadamente $2 \mathrm{~mm}$ de diámetro; posteriormente, se utilizó un molino ciclónico (Cyclotec ${ }^{\mathrm{TM}}$ 1093, FOSS Tecator, Hoganas, Sweden) con una malla de acero inoxidable de $0.5 \mathrm{~mm}$ de diámetro.

\section{Determinación de antocianinas totales}

El método utilizado se basó en el protocolo descrito por Galicia et al. (2012). Para la extracción de antocianinas las muestras previamente molidas se secaron a $65^{\circ} \mathrm{C}$ por 16 $\mathrm{h}$ a fin de asegurar una humedad homogénea de las mismas. De cada muestra de harina se pesaron $20 \mathrm{mg}$ y se colocaron en un tubo Eppendorf, al que se agregaron 1.5 $\mathrm{mL}$ de ácido trifluoroacético (TFA) al $1 \%$. Los tubos se agitaron en el vortex (Scientific Industries, Genie-2, New York, USA), luego fueron incubados horizontalmente sobre hielo y en agitación a $150 \mathrm{rpm}$ por $90 \mathrm{~min}$. Terminado el tiempo de incubación, las muestras se centrifugaron a 15,339 X g durante $5 \mathrm{~min}$. De éstas se transfirieron por duplicado 200 $\mu \mathrm{L}$ del sobrenadante de cada muestra a los pozos de una microplaca y se leyó la absorbancia a $520 \mathrm{~nm}$ en un espectrofotómetro de microplacas BioTek® ( $\mu$ Quant MQX200, Winooski, Vermont, USA). Se elaboró una curva patrón de cloruro de pelargonidina de 0 a $15 \mu \mathrm{gL}^{-1}$ (Sigma-Aldrich Cat. P-1659-10MG CAS 134-04-3) para realizar la estimación del contenido de antocianinas. Los resultados se expresaron en $\mu \mathrm{g}$ de pelargonidina (Pel) por g de muestra en peso seco (PS).

\section{Cuantificación de antocianinas glicosiladas}

A partir de las 554 muestras previamente molidas se hizo una selección de las 126 con mayor contenido de antocianinas totales determinadas por espectroscopía de UV-Vis para su análisis en HPLC. El análisis del perfil 
Cuadro 1. Razas asociadas a las 254 accesiones de maíces pigmentados utilizadas en la cuantificación.

\begin{tabular}{|c|c|c|}
\hline Área de adaptación & Accesiones & Raza \\
\hline Valles altos & 124 & $\begin{array}{l}\text { Azul, Cristalino Chihuahua, Elotes Occidentales, Vandeño, Elotes Cónicos, } \\
\text { Bolita, Negro de Chimaltenango, Celaya, Cónico y Chalqueño. }\end{array}$ \\
\hline
\end{tabular}

de antocianinas y su cuantificación mediante HPLC fueron realizados en un cromatógrafo de líquidos con detector de arreglo de diodos de la marca Alliance Waters (Waters ${ }^{\circledR}$ 2696, Milford, Massachusetts, USA), a través del método descrito por Salinas et al. (2012a) con ligeras modificaciones. Las condiciones cromatográficas fueron: una fase móvil de agua con 0.09 \% de TFA a un pH de 2.0, como solvente $A$, y una mezcla de acetonitrilo-metanol (50:50) con $0.09 \%$ de TFA, como solvente B. Se utilizó una columna Zorbax SB Aq $3.5 \mu \mathrm{m} 4.6$ × 150 mm (No. Parte: 863953914) y una pre-columna SecurityGuard ${ }^{\mathrm{Tm}}$ cartridge kit Phenomenex® (No. Parte: KJO-4282). El tiempo de elución fue de 20 min a un flujo de $0.4 \mathrm{~mL} \mathrm{~min}^{-1}$ y la temperatura de la columna fue de $35^{\circ} \mathrm{C}$.

El volumen de inyección fue de $20 \mu \mathrm{L}$. Las antocianinas fueron detectadas a una longitud de onda de $520 \mathrm{~nm}$. Los estándares utilizados fueron cianidina 3-glucósido (Sigma-Aldrich, Cat. 52976 pureza $\geq 95 \%$ ), pelargonidina 3-glucósido (Sigma-Aldrich, Cat. 79576 pureza $\geq 97 \%$ ) y peonidina 3-glucósido (Sigma-Aldrich, Cat. 40796 pureza $\geq 95 \%$ ); cada estándar fue reconstituido con $5 \mathrm{~mL}$ de metanol con ácido clorhídrico al 1 \% para obtener una concentración de $200 \mu \mathrm{g} \mathrm{mL} \mathrm{mL}^{-1}$; posteriormente, se agregó a cada uno $200 \mu \mathrm{L}$ de metanol acidificado $(\mathrm{pH} 2)$. De cada estándar se tomó $1 \mathrm{~mL}$ de la solución de $200 \mu \mathrm{g} \mathrm{mL} \mathrm{m}^{-1}$ y se adicionó $1 \mathrm{~mL}$ de metanol acidificado para tener una solución stock de $100 \mu \mathrm{g} \mathrm{mL}^{-1}$ y se procedió a la realización de la curva de calibración, en un rango de concentraciones de 1 a $100 \mu \mathrm{g} \mathrm{mL}$.

\section{Espectroscopía de infrarrojo cercano (NIR). Obtención de espectros}

La misma harina de las 554 muestras analizadas por espectroscopía de UV-Vis fue utilizada para desarrollar los modelos de calibración de NIR. La humedad se ajustó entre 10 y $11 \%$, y se escanearon de 2 a 4 g por muestra en un equipo NIRS ${ }^{\text {m }} 6500$ (monocromador FOSS NIRSystems, Inc., Silver Spring, Maryland, USA) el cual registra las señales de reflectancia difusa, mediante celdas circulares (diámetro interno de $35 \mathrm{~mm}$ y profundidad de $8 \mathrm{~mm}$ ). Los espectros entre 400 y $2500 \mathrm{~nm}$ de longitud de onda fueron capturados. Se registró el valor promedio de log (1/R) de 400 a $2500 \mathrm{~nm}$ a intervalos de $2 \mathrm{~nm}$ como un promedio de 32 sub escaneos, donde $\mathrm{R}$ fue el valor de reflectancia a cada longitud de onda. Para asegurar que tanto las muestras de la calibración como las de la validación independiente estuvieran dentro de los mismos intervalos del contenido de antocianinas, las 554 muestras se ordenaron ascendentemente de acuerdo con la concentración de dichos pigmentos y se fueron seleccionando dos para los modelos de calibración y una para la validación. Así, 371 muestras se utilizaron para la realización de las calibraciones y 183 muestras como grupo independiente para validar los modelos.

\section{Pre-tratamiento de los espectros y procedimientos matemáticos para las calibraciones y validación}

Las ecuaciones de calibración fueron generadas mediante el software WinISI ${ }^{\mathrm{T} M}$ versión 4.5.0.14017 de Infrasoft International (FOSS NIRSystems, Inc., Silver Spring, Maryland, USA), a través del modelo de regresión lineal de mínimos cuadrados parciales modificado (MPLS, por sus siglas en inglés) y técnicas de validación cruzada. Previo a la regresión MPLS fueron aplicados tratamientos matemáticos y de derivación, de los que se registraron los mejores resultados con las combinaciones 0, 0, 1, 1 y 1, 4, 4, 1 ; donde, el primer número corresponde al grado de derivación, el segundo número es el espacio entre cada punto de los datos, y el tercero y cuarto son los puntos utilizados para el suavizado. Adicionalmente, un algoritmo de ajuste Detrend fue aplicado, el cual consiste en restar la media o la línea de mayor ajuste de los datos para reducir los efectos de radiación dispersa.

La desviación estándar (SDc) y el error estándar de laboratorio (SEL) de las muestras utilizadas para la calibración fueron calculados, y se reportó el porcentaje de muestras anómalas que fueron omitidas para la realización de las calibraciones (valores atípicos), además de los términos 
utilizados en los modelos con la regresión lineal de mínimos cuadrados parciales modificados. Los coeficientes de determinación de la calibración $\left(R^{2} \mathrm{C}\right)$ y de la validación independiente $\left(R^{2} v i\right)$ fueron calculados, además de la desviación estándar del grupo de validación independiente (SDgvi) y de los valores obtenidos en la validación para cada modelo (SDvi).

Adicionalmente, el cociente de la desviación de desempeño (Radio Performance Deviation, RPD, por sus siglas en inglés) fue determinado tras dividir SDvi entre el SEP (Error Estándar de Predicción), ya que la calidad y robustez de la calibración NIRS también puede ser evaluada con este parámetro; un valor menor a 2 indicaría una calibración poco confiable (Chang et al., 2001).

Adicional a los análisis estadísticos descritos anteriormente, para la elaboración y validación de los modelos para el NIR, se utilizó el lenguaje y ambiente para cómputo estadístico R ( $R$ Core Team, 2015) para determinar los coeficientes de correlación $r$ de Pearson de los métodos de cuantificación de antocianinas.

\section{RESULTADOS Y DISCUSIÓN}

\section{Antocianinas totales}

El contenido de antocianinas totales de las muestras osciló entre 2.52 y $1989.97 \mu^{\prime}$ Pel g-1 PS (Figura 1), con un promedio de $281.01 \mu \mathrm{g}$ Pel g $\mathrm{g}^{-1}$ PS. Lo anterior muestra una alta variabilidad dentro de las muestras analizadas, con contenidos superiores a los reportados previamente en grano de maíz (Camelo-Méndez et al., 2016; Espinosa et al., 2009; Salinas-Moreno et al., 2012b; Urias-Lugo et al., 2015; Žilić et al., 2012). Diez muestras superaron los 1000 $\mu \mathrm{g}$ Pel $\mathrm{g}^{-1} \mathrm{PS}$, en las que destacaron las accesiones de Tlaxcala C11-IXT, C08-IXT y C10-IXT, con concentraciones de 1989.97, 1952.49 y $1741.89 \mu \mathrm{g}$ Pel g ${ }^{-1}$ PS, respectivamente. Salinas et al. (2012b) reportaron el contenido de antocianinas en 18 muestras recolectadas en el Estado de México, Ciudad de México y Oaxaca, con valores promedio de 579.4 a 1046.1, 997.8 a 1332.2 y 304.1 a $528.0 \mu \mathrm{g} \mathrm{Pel}$ $\mathrm{g}^{-1}$ PS para las razas Chalqueño, Elotes Cónicos y Bolita, respectivamente. En el presente estudio se encontraron contenidos más bajos para las razas Chalqueño, Elotes Cónicos y Bolita, con 256.3, 336.4 y 89.7 a 242.0 g Pel g-1 $\mathrm{PS}$, respectivamente.

\section{Perfil de antocianinas glicosiladas}

Cianidina 3-glucósido, pelargonidina 3-glucósido y peonidina 3-glucósido fueron las tres antocianinas glicosiladas observadas en el perfil de HPLC (Cuadro 2). Cianidina 3-glucósido fue la antocianina encontrada en mayor cantidad, con una media de $154.6 \mathrm{\mu g} \mathrm{g}^{-1}$ PS, lo que coincide con reportes previos en granos de maíz púrpura y magenta (Salinas et al., 2013; Salinas-Moreno et al., 2003). La accesión de Tlaxcala C11-IXT presentó $1989.9 \mu \mathrm{g}$ Pel g-1 PS de antocianina total, con una distribución de $28.1 \%$ de cianidina 3-glucósido, $1.0 \%$ de pelargonidina 3-glucósido y $7.1 \%$ de peonidina 3-glucósido. La muestra perteneciente a la accesión BOZM342, color púrpura muy intenso, destacó en el contenido de cianidina 3-glucósido, con una concentración de $628.3 \mu \mathrm{g} \mathrm{g}^{-1}$ PS (Figura 2). Estos resultados sugieren un potencial para estos maíces como fuentes de nutracéuticos. Se ha reportado, por ejemplo,

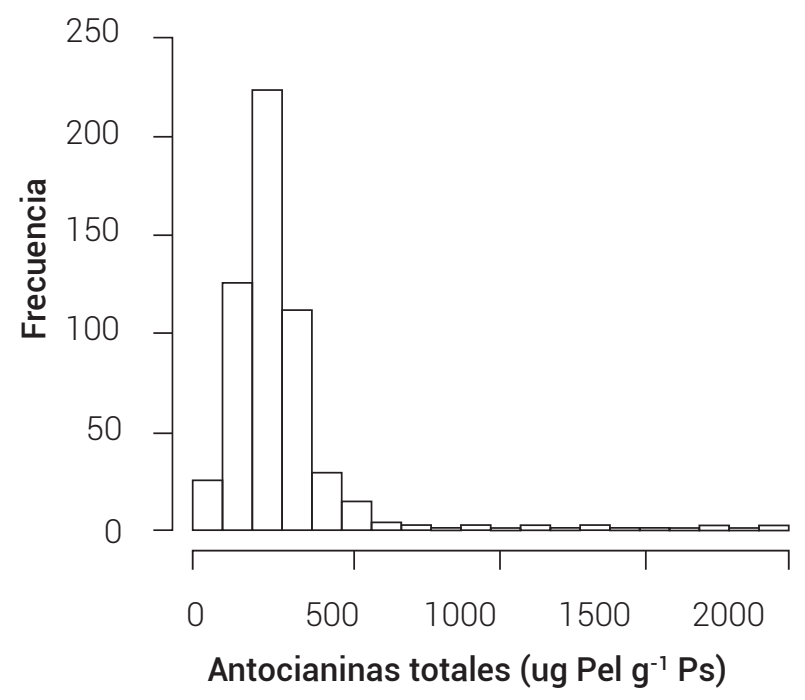

Figura 1. Distribución del contenido de antocianinas totales por espectroscopía de UV-Vis en 554 muestras de maíz pigmentado. 
que cianidina-3-glucósido ayuda al tratamiento contra la inflamación intestinal y se ha demostrado que esta antocianina es eficaz para la inhibición de las marcadoras proinflamatorias producidas por citoquinas (Serra et al., 2013).

\section{Modelos de calibración de NIR}

Se realizaron 32 calibraciones para el NIR, de las cuales cuatro tuvieron un $R^{2} \mathrm{C}$ superior a 0.7. El porcentaje de datos atíícos eliminados con los criterios dados en las calibraciones fue bajo (de 3.5 a $5.1 \%$ ), lo cual sugiere que las ecuaciones son robustas. En la Cuadro 3 se presentan los resultados de las dos mejores calibraciones llamadas at04 y at22. Los coeficientes de determinación de validaciones cruzadas fueron ligeramente menores con respecto a los $R^{2} c, 0.64$ y 0.65 , para at04 y at 22 , respectivamente.

Se realizó una validación independiente con 183 muestras. Los coeficientes de determinación obtenidos de 0.7698 y 0.7758 (Figura 3), para at04 y at22, respectivamente, fueron superiores a los obtenidos en la validación interna y similares a coeficientes de determinación obtenidos en la validación de ecuaciones NIR para la cuantificación de contenido total de antocianinas (TAC) en flores y frutos (Cavalcanti et al., 2013; Xiaowei et al., 2014); para maíz no se encontraron reportes. El intervalo de predicción abarcó el 74.9 y $78.2 \%$ del calculado en el grupo de validación (Cuadro 3); el porcentaje que no está dentro del intervalo es debido a las muestras con los valores más altos de TAC, las cuales fueron identificadas como valores atípicos. Adicionalmente, los valores RPD obtenidos (2.9 y 3.0) indi- can que los modelos son lo suficientemente robustos para monitorear el contenido total de antocianinas en muestras de maíz, especialmente para apoyar las etapas iniciales de los programas de mejoramiento (Cuadro 3). Estos resultados de los modelos de calibración son similares a los obtenidos para la cuantificación de lisina y triptófano en muestras de harina de maíz blanco (Rosales et al., 2011) y que actualmente se utilizan para apoyar los programas de mejoramiento de maíz de alta calidad proteica (TwumasiAfriyie et al., 2016).

\section{Correlación entre los métodos utilizados para la cuantificación de antocianinas}

Correlaciones significativamente altas fueron obtenidas entre los datos de antocianinas totales obtenidos por el NIR y los obtenidos por espectroscopía de UV-Vis y HPLC, de 0.825 y 0.827 , respectivamente, lo que confirma la utilidad del método NIR para la cuantificación de antocianinas en maíces pigmentados.

\section{CONCLUSIONES}

Los dos modelos propuestos para NIR son robustos dados los valores de SEP, SDvi, R²vi y RPD obtenidos. Estos modelos de NIR para antocianina brindan una alternativa rápida y de bajo costo para la determinación de antocianinas totales. Los resultados obtenidos en los métodos de análisis de antocianinas estuvieron altamente correlacionados entre sí. El método por HPLC es una herramienta muy importante para la caracterización y cuantificación de

Cuadro 2. Cuantificación por HPLC de antocianinas glicosiladas.

\begin{tabular}{lccc}
\hline Antocianina & Promedio $\left(\mu \mathrm{g} \mathrm{g}^{-1} \mathrm{PS}\right)$ & Valor máximo $\left(\mu \mathrm{g} \mathrm{g}^{-1} \mathrm{PS}\right)$ & Valor mínimo $\left(\mu \mathrm{g} \mathrm{g}^{-1} \mathrm{PS}\right)$ \\
\hline Cianidina 3-glucósido & 154.69 & 628.32 & 24.10 \\
Pelargonidina 3-glucósido & 14.33 & 521.14 & 0.62 \\
Peonidina 3-glucósido & 25.45 & 158.83 & 10.37 \\
\hline
\end{tabular}

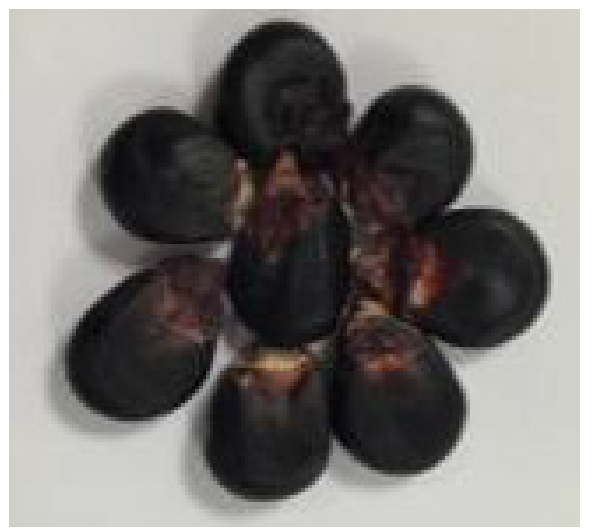

Figura 2. Granos de color púrpura en la accesión BOZM342. 
Cuadro 3. Coeficiente de determinación y características de los modelos aplicados para la determinación de antocianinas totales.

\begin{tabular}{|c|c|c|c|c|c|c|c|c|c|c|}
\hline \multirow{2}{*}{ Modelo } & \multicolumn{5}{|c|}{ Grupo de calibración } & & \multirow{2}{*}{\multicolumn{2}{|c|}{$\%$ Valores atípicos ${ }^{\bullet}$ MPLS terms ${ }^{\bullet}$}} & \multirow[b]{2}{*}{$\mathrm{R}^{2} \mathrm{C}^{\mathrm{x}}$} & \\
\hline & $\mathrm{N}$ & Intervalo & Promedio & $\mathrm{SDC}^{\dagger}$ & $\mathrm{SEL}^{+\dagger}$ & & & & & \\
\hline at04 & & & & & & & 3.5 & 11 & 0.7068 & \\
\hline at22 & 371 & $2.5-1989.9$ & 290.6 & 204.8 & 19.9 & & 5.1 & 9 & 0.7757 & \\
\hline \multirow{2}{*}{ Modelo } & \multicolumn{4}{|c|}{ Grupo de validación } & \multicolumn{6}{|c|}{ Validación independiente } \\
\hline & $\mathrm{N}$ & Intervalo & Promedio & SDgvixx & Intervalo & Promedio & SDviy & $\mathrm{R}^{2} \mathrm{vi} \mathrm{i}^{\mathrm{y}}$ & SEPz & $\mathrm{RPD}^{z z}$ \\
\hline at04 & & & & & $0-925.1$ & 266 & 115.90 & 0.7698 & 39.90 & 2.90 \\
\hline at22 & 183 & $3.9-1237.7$ & 280.6 & 153.50 & $0-964.8$ & 266.8 & 120.10 & 0.7758 & 39.90 & 3.01 \\
\hline
\end{tabular}

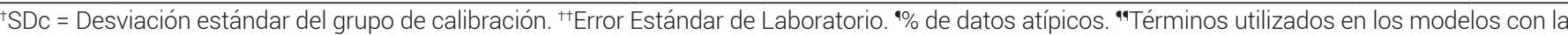
regresión lineal de mínimos cuadrados parciales modificados. ${ }^{\times}{ }^{2} \mathrm{C}=$ Coeficiente de determinación de la calibración. ${ }^{\times \times}$SDgvi = Desviación estándar del grupo de validación independiente. ${ }^{y}$ SDvi = Desviación estándar de la validación independiente. ${ }^{y} R^{2}$ vi = Coeficiente de determinación de la validación independiente. ${ }^{\mathrm{zSEP}}=$ Error estándar de predicción. ${ }^{\mathrm{z} R P D D}$ = Cociente de la Desviación de Desempeño, calculado como SDvi/SEP.
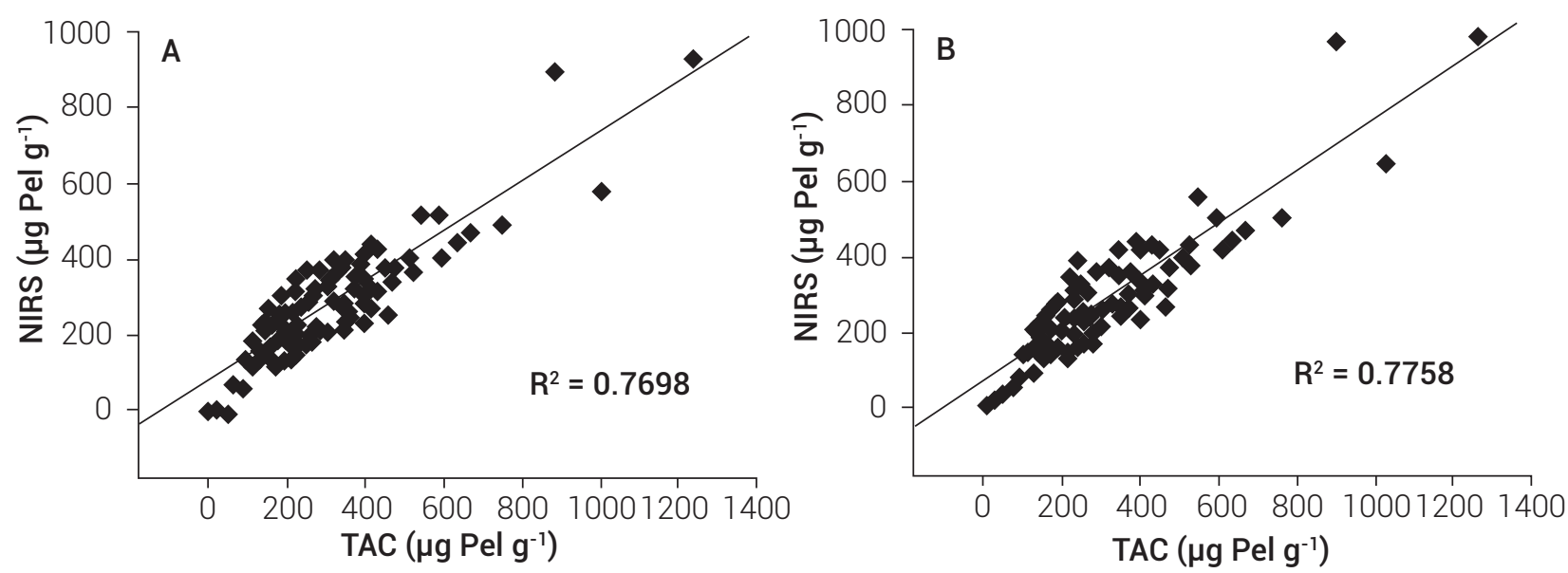

Figura 3. Regresiones lineales de TAC y NIRS de 183 muestras, calibración at04 (A) y at22 (B).

antocianinas individuales; sin embargo, su alto costo, necesidad de equipo y personal especializado hacen que sea un método más complejo comparado con el NIR. Todas las muestras analizadas presentaron cianidina-3-glucósido, pelargonidina-3-glucósido y peonidina-3-glucósido en el perfil de HPLC, en la que cianidina-3-glucósido fue la antocianina encontrada en mayor cantidad.

\section{AGRADECIMIENTOS}

Este trabajo ha sido parcialmente cubierto por el programa "MASAGRO: Modernización sustentable de la agricultura tradicional" y por Kellogg's. El primer autor es apoyado por una beca de doctorado de CONACYT en el Postgrado de Recursos Genéticos y Productividad - Genética (COLPOS Campus Montecillo). Agradecemos a los Drs. Ricardo
Ernesto Preciado Ortiz, Gilberto Esquivel Esquivel, Víctor Antonio Vidal Martínez, Noel Orlando Gómez Montiel, MSc. Arturo Daniel Terrón Ibarra, Humberto L. Vallejo Delgado, Armando Guadarrama Espinoza, Enrique Rodríguez Chanona, Daniel Chepetla Calderón, Javier Manjarrez Juárez y a todo el equipo involucrado en hacer polinizaciones a mano para formar el material usado en el experimento en las estaciones experimentales del Instituto Nacional de Investigaciones Forestales, Agrícolas y Pecuarias (INIFAP), Productores de Semillas de Copandaro e Investigación y Capacitación Agropecuaria (ICAMEX). Agradecemos a los Drs. Terrance Molnar y Sarah Hearne y a el M. C. Daniel Díaz Mora y a todo el personal del laboratorio de calidad de maíz del CIMMYT por su colaboración, muestras de maíz de Tlaxcala e información de pasaporte del material utilizado. 


\section{BIBLIOGRAFÍA}

Agama-Acevedo E., Y. Salinas-Moreno, G. Pacheco-Vargas y L. A. Bello-Pérez (2011) Características físicas y químicas de dos razas de maíz azul: morfología del almidón. Revista Mexicana de Ciencias Agrícolas 2:317-329.

Ananga A., V. Georgiev, J. Ochieng, B. Phills and V. Tsolova (2013) Production of anthocyanins in grape cell cultures: a potential source of raw material for pharmaceutical, food, and cosmetic industries. In: The Mediterranean Genetic Code - Grapevine and Olive. D. Poljuha and B. Sladonja (eds.). InTech. Rijeka, Croatia. pp:247287.

Camelo-Méndez G. A., M. J. Jara-Palacios, M. L. Escudero-Gilete, B. Gordillo, D. Hernanz, O. Paredes-López, P. E. Vanegas-Espinoza, A. A. Del VillarMartínez and F. J. Heredia (2016) Comparative study of phenolic profile, antioxidant capacity, and color-composition relation of roselle cultivars with contrasting pigmentation. Plant Foods for Human Nutrition 71:109-114.

Cavalcanti I. M. R., K. M. Gomes L., V. Garcia L., J. D. Cruz P. and G. H. de Almeida T. (2013) Total anthocyanin content determination in intact açaí (Euterpe oleracea Mart.) and palmitero-juçara (Euterpe edulis Mart.) fruit using near infrared spectroscopy (NIR) and multivariate calibration. Food Chemistry 136:1160-1164.

Chang C. W., D. A. Laird, M. J. Mausbach and C. R. Hurburgh (2001) Nearinfrared reflectance spectroscopy - principal components regression analyses of soil properties. Soil Science Society of America Journal 65:480-490

CONABIO, Comisión Nacional para el Conocimiento y Uso de la Biodiversidad (2016) Razas de maíz en México. http://www.biodiversidad.gob. $\mathrm{mx} / \mathrm{usos} / \mathrm{maices} / \mathrm{razas} 2012 \mathrm{html}$ (Mayo 2016).

Espinosa T. E., M. C. Mendoza C., F. Castillo G., J. Ortiz C., A. Delgado A. y A. Carrillo S. (2009) Acumulación de antocianinas en pericarpio y aleurona del grano y sus efectos genéticos en poblaciones criollas de maíz pigmentado. Revista Fitotecnia Mexicana 32:303-309

Fernández S. R., L. A. Morales C. y A. Gálvez M. (2013) Importancia de los maíces nativos de México en la dieta nacional. Una revisión indispensable. Revista Fitotecnia Mexicana 36:275-283.

Galicia L., A. Miranda, M. G. Gutiérrez, O. Custodio, A. Rosales, N. Ruíz, R. Surless y N. Palacios (2012) Protocolos de Laboratorio 2012. Laboratorio de Calidad Nutricional de Maíz y Análisis de Tejido Vegetal. CIMMYT. México, D.F. 50 p.

He J. and M. M. Giusti (2010) Anthocyanins: natural colorants with health-promoting properties. Annual Review of Food Science and Technology 1:163-187.

Hellin J., A. Keleman, D. López, L. Donnet y D. Flores (2013) La importancia de los nichos de mercado. Un estudio de caso del maíz azul y del maíz para pozole en México. Revista Fitotecnia Mexicana 36:315-328.

Hosoda K., M. Miyaji, H. Matsuyama, S. Haga, H. Ishizaki and K. Nonaka (2012) Effect of supplementation of purple pigment from anthocyaninrich corn (Zea mays L.) on blood antioxidant activity and oxidation resistance in sheep. Livestock Science 145:266-270.
R Core Team (2015) R: A language and environment for statistical computing. R Foundation for Statistical Computing, Vienna, Austria http://www.R-project.org/ (Marzo 2016).

Rosales A., L. Galicia, E. Oviedo, C. Islas and N. Palacios-Rojas (2011) Nearinfrared reflectance spectroscopy (NIRS) for protein, tryptophan, and lysine evaluation in quality protein maize (QPM) breeding programs. Journal of Agricultural and Food Chemistry 59:10781-10786.

Salinas-Moreno Y., F. Martínez-Bustos, M. Soto-Hernández, R. Ortega-Paczka y J. L. Arellano-Vázquez (2003) Efecto de la nixtamalización sobre las antocianinas del grano de maíces pigmentados. Agrociencia 37:617-628.

Salinas M. Y., F. J. Cruz C., S. A. Díaz O. y F. Castillo G. (2012a) Granos de maíces pigmentados de Chiapas, características físicas, contenido de antocianinas y valor nutracéutico. Revista Fitotecnia Mexicana 35:33-41

Salinas-Moreno Y., J. J. Pérez-Alonso, G. Vázquez-Carrillo, F. Aragón-Cuevas y G. A. Velázquez-Cardelas (2012b) Antocianinas y actividad antioxidante en maíces (Zea mays L.) de las razas Chalqueño, Elotes Cónicos y Bolita. Agrociencia 46:693-706.

Salinas M. Y., C. García S., B. Coutiño E. y V. A. Vidal M. (2013) Variabilidad en contenido y tipos de antocianinas en granos de color azul/ morado de poblaciones mexicanas de maíz. Revista Fitotecnia Mexicana 36:285-294.

Serra D., J. Paixão, C. Nunes, T. C. P. Dinis and L. M. Almeida (2013) Cyanidin-3-glucoside suppresses cytokine-induced inflammatory response in human intestinal cells: comparison with 5-aminosalicylic acid. PLOS ONE 8:e73001, doi:10.1371/journal. pone.0073001

SIAP, Sistema de Información Agroalimentaria y Pesquera (2017) Agricultura: Producción Anual: Cierre de la producción agrícola por cultivo. SAGARPA. México. http://infosiap.siap.gob.mx/aagricola_siap_gb/ientidad/index.jsp (Marzo 2017).

Twumasi-Afriyie S., N. Palacios-Rojas, D. Friesen, A. Teklewold, D. Wegary, H. De Groote and B. M. Prasanna (2016) Guidelines for the Quality Control of Quality Protein Maize (QPM) Seed and Grain. Technical Bulletin. CIMMYT. Addis Ababa, Ethiopia. 38 p.

Urias-Lugo D. A., J. B. Heredia, M. D. MuyRangel, J. B. Valdez-Torres, S. 0 Serna-Saldivar and J. A. Gutiérrez-Uribe (2015) Anthocyanins and phenolic acids of hybrid and native blue maize (Zea mays L.) extracts and their antiproliferative activity in mammary (MCF7) Liver (HepG2), colon (Caco2 and HT29) and prostate (PC3) cancer cells. Plant Foods for Human Nutrition 70:193-199.

Xiaowei H., Z. Xiaobo, Z. Jiewen, S. Jiyong, Z. Xiaolei and M. Holmes (2014) Measurement of total anthocyanins content in flowering tea using near infrared spectroscopy combined with ant colony optimization models. Food Chemistry 164:536-543.

Zhang Y., S. K. Vareed and M. G. Nair (2005) Human tumor cell growth inhibition by nontoxic anthocyanidins, the pigments in fruits and vegetables. Life Sciences 76:1465-1472.

Žilić S., A. Serpen, G. Akıllıolu, V. Gökmen and J. Vančetović (2012) Phenolic compounds, carotenoids, anthocyanins, and antioxidant capacity of colored maize (Zea mays L.) kernels. Journal of Agricultural and Food Chemistry 60:1224-1231. 
